\title{
SaS noise suppression for OFDM wireless communication in rayleight channel
}

\author{
Heyem Hamlili, Samir Kameche, Abdelhafid Abdelmalek \\ STIC Laboratory, Department of Telecommunication, Abou Bakr Belkaid University, Algeria
}

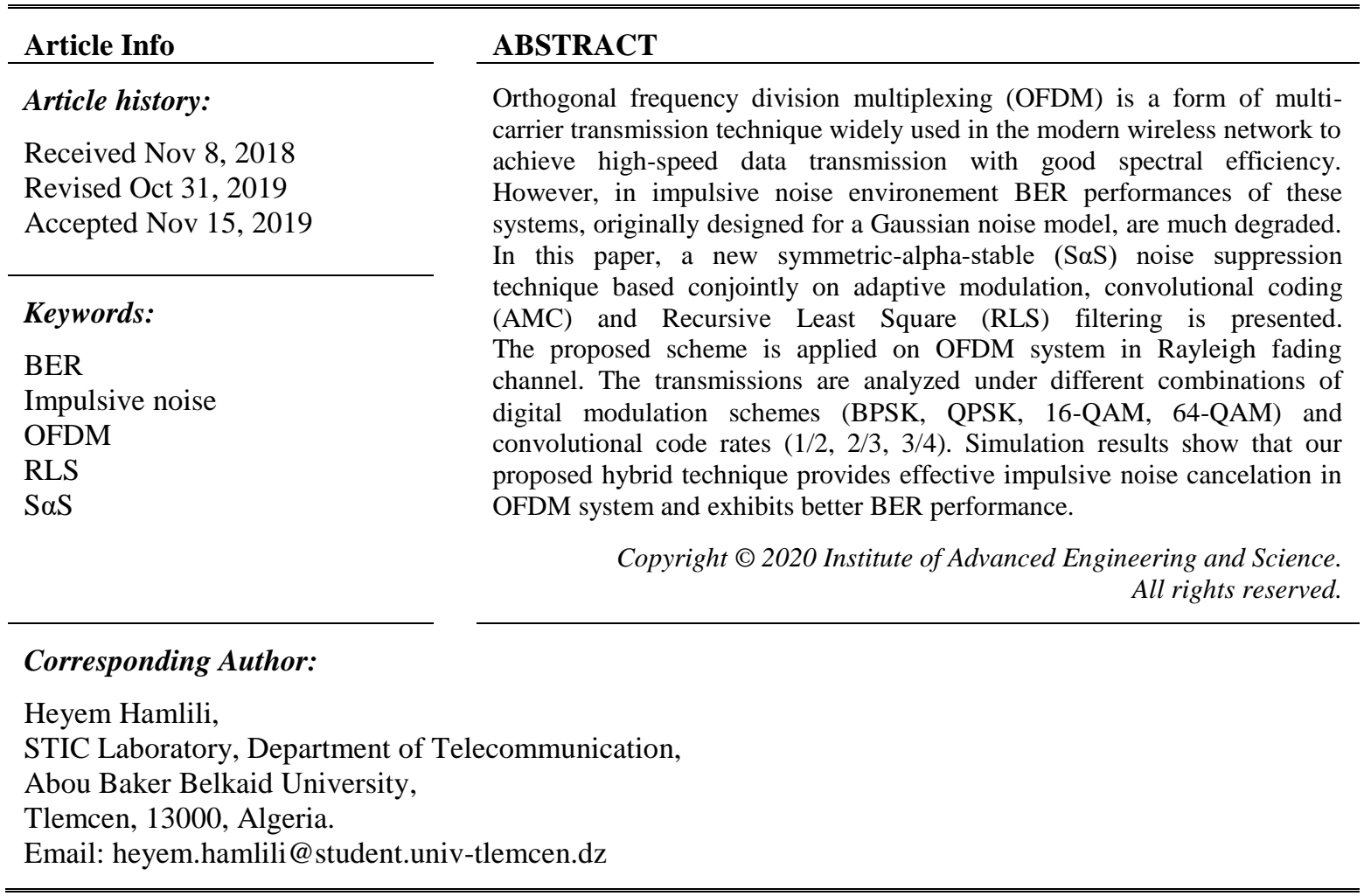

\section{INTRODUCTION}

In the communication system, we can not avoid the existence of disturbances that may occur during the communication. Traditional methods treat noise as Gaussian white noise AWGN in which its statistical and spectral characteristics are predefined. However, this is not the case practically the noise is non-Gaussian impulsive in nature, which is generally nonstationary with a very complex frequency behavior. We find this kind of noise in underwater communications [1], highfrequency communications, LPC broadband communications [2] and telemedicine [3] etc.In the literature, several models have been proposed to simulate impulsive noise Middleton class A [4], Bernoulli Gaussian [5] distribution or symmetric alpha-stable distribution $(\mathrm{S} \alpha \mathrm{S})[6]$.We consider the $\alpha$-stable noise model for our study.

Improvements in theOrthogonal Frequency Division Multiplexing OFDM communication system have become a major focus of research, as itis increasingly being adopted as a physical-layer modulation scheme in latest and emerging wireless standards. OFDM has been chosen for Wi-Fi arena [7] where the standards like 802.11a, 802.11n, 802.11ac and more. It has also been adopted for the cellular telecommunications standard LTE / LTE-A, WiMAX [8] and many more. Many approaches confirm that an OFDM family is the right candidate for 5G like W-OFDM [9], G-DFT-s-OFDM [10], WR-OFDM [11] and F-OFDM [12].

OFDM systems are usually corrupted by an impulsive noise which has more harmful effects. The performance of the OFDM system is degraded and the efficiency of the transmission is reduced due impulsive noise's broad frequency component. Nowadays, the main concern of this research area is to investigate a new method to mitigate this type of noise, therefore improving systems performance in terms of 
bit error rate. Various techniques are described in the literature to eliminate the impulsive noise of the transmitted original signal. The conventional method of suppressing this type of noise is the median filter with some signal degradation [13]. Wavelet transform based logarithmic shrinkage technique is used in [14] to eliminate the impulsive noise of corrupted images. Adaptive filters have been used successfully for the same purpose. A technique based on trained Least Mean Square algorithm (LMS) adaptive filtering is proposed by Khedkar [15] to mitigate the effect of inter-carrier interference. LMS is also used in [16] to reduce the effect of periodic impulsive noise in the Power Line Communication channel. Alina Mirza et al. in [17] used the State Space Recursive Least Square (SSRLS) algorithm to reduce the impulsive noise in the OFDM system. Srinu Pyla et al. [18] analyse the performance of adaptive filter channel estimated MIMO OFDM communication system. However, the proposed solutions are still suboptimal and further improvements are required. Thus, the goal of this research is to improve the performance of OFDM systems by minimizing $(\mathrm{S} \alpha \mathrm{S})$ impulse noise in Rayleigh Fading Channel, using a new hybrid approach based on adaptive RLS filters and adaptive modulation and convolutional coding (AMC) technique applied to OFDM sub-channels.

The paper is organized as follows: section 2 discusses impulsive noise model, where precesily (SaS) distribution and geometric signal-to-noise-ration (GSNR) are presented. Section 3 briefly explain the adopted OFDM system. Section 4 describes our proposed solution for imulpsive noise cancellation, based conjointly on adaptative RLS filter and AMC technique. Simulation results and discussion are presented in section 5. Section 6 concludes the paper.

\section{IMPULSIVE NOISE MODEL}

\subsection{SaS distribution}

In this work, as pointed before we consider $\alpha$-stable distribution model $[19,20]$. A real random variable $\mathrm{X}$ follows a law $\alpha$-stable if and only if its characteristic function is described as follows:

$$
\Psi_{\alpha}(\mathrm{t})=\exp \left\{-\gamma^{\alpha}|\mathrm{t}|^{\alpha}[1+\mathrm{i} \beta \operatorname{sign}(\mathrm{t}) \omega(\mathrm{t}, \alpha)]+\mathrm{i} \delta \mathrm{t}\right\}
$$

Where

$$
\begin{aligned}
& \omega(t, \alpha)=\left\{\begin{array}{c}
-\tan \left(\frac{\pi \alpha}{2}\right) \text { si } \alpha \neq 1 \\
\frac{2}{\pi} \ln |t| \text { si } \alpha=1
\end{array}\right. \\
& \text { And } \operatorname{sign}(t)=\left\{\begin{array}{c}
1 \text { si } t>0 \\
0 \text { si } t=0 \\
-1 \text { si } t<0
\end{array}\right.
\end{aligned}
$$

An $\alpha$-stable distribution is completely defined by four parameters summarized in Table 1 and it can be denoted $\mathrm{X} \sim \mathrm{S}_{\alpha}(\beta, \gamma, \delta)$.

Table 1. Parameter descriptions for stable distributions

\begin{tabular}{ccc}
\hline Parameters & Symbol & Values \\
\hline Characteristic exponent & $\alpha$ & $\alpha \in] 0,2]$ \\
Scale parameter & $\gamma$ & $\gamma>0$ \\
Location parameter & $\delta$ & $\delta \in]-\infty,+\infty[$ \\
The symmetry parameter & $\beta$ & $\beta \in[-1,1]$ \\
\hline
\end{tabular}

A random variable is symmetric $\alpha$-stable $(\mathrm{S} \alpha \mathrm{S})$ if $\beta$ and $\gamma$ are equal to zero [21], subsequently the distribution of such a variable reduces to $\mathrm{X} \sim \mathrm{S}_{\alpha}(0,0, \delta)$ if:

- $\quad \alpha=2$ the distribution reduces to a Gaussian distribution described by the following Probability Density Function (PDF):

$$
f(x)=\frac{1}{\sqrt{4 \pi \gamma^{2}}} \exp \left(-\frac{x^{2}}{2 \gamma^{2}}\right)
$$

- $\quad \alpha=1$ we have a Cauchy distribution where the PDF is defined as follows:

$$
f(x)=\frac{\gamma}{\pi\left(\gamma^{2}+x^{2}\right)}
$$


- $\quad \alpha=1 / 2$ The distribution follow the Low of Lévy and the PDF is presented as follows:

$$
f(x)=\sqrt{\frac{\gamma}{2 \pi}} \frac{1}{(x-\delta)^{\frac{3}{2}}} \exp \left(-\frac{\gamma}{2(x-\delta)}\right), \delta<x<\infty
$$

For the other cases we can approach PDF of a stable law by the inverse transform of the characteristic function. The integral is written as follows:

$$
f_{X}(x)=\frac{1}{2 \pi} \int_{-\infty}^{\infty} e^{-i t x} \Psi_{\alpha}(t) d t
$$

Figure 1 shows PDFs of the SaS model for different values of $\alpha$ while the other parameters are kept fixed at 0 .

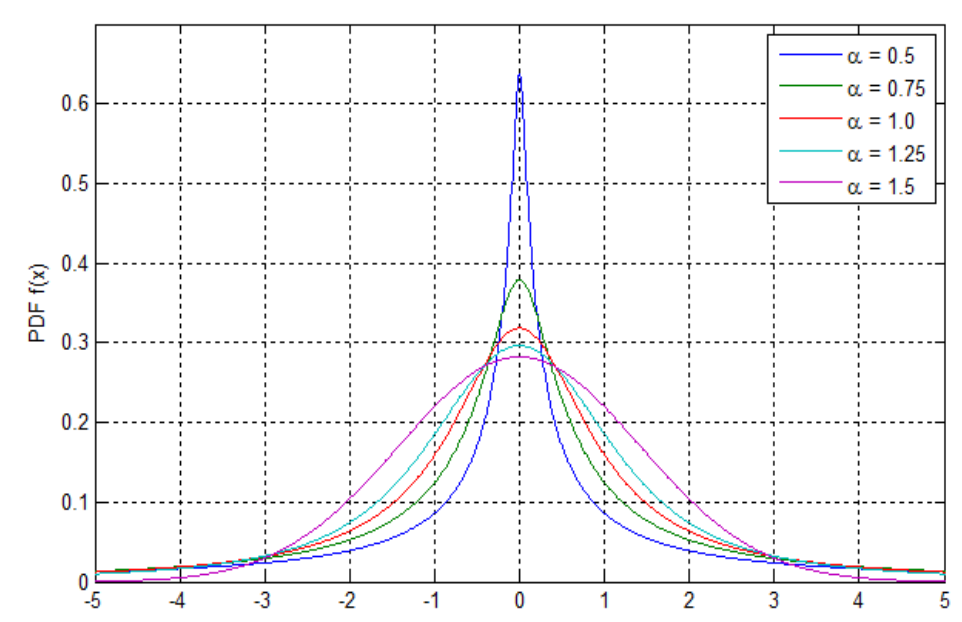

Figure 1. S $\alpha$ S distributions for different values of $\alpha$

\subsection{GSNR}

For digital communication systems in impulse noise environments the BER curve is conventionally represented as a function of the GSNR geometric signal-to-noise ratio which was first proposed by Gonzalez in [22]. The GSNR is defined as:

$$
G S N R=\frac{1}{2 C_{g}} \cdot\left(\frac{A}{S_{0}}\right)^{2}
$$

Where:

- $\quad \mathrm{S}_{0}$ is the geometric power of a symmetric $\alpha$-stable given by :

$$
S_{0}=S_{0}(X)=e^{2 E(\ln |x|)}
$$

- $\quad \mathrm{C}_{\mathrm{g}}$ is the exponential of the Euler constant $C_{g}=e^{C_{e}}=1.7811$

- $\quad \mathrm{A}$ is the peak amplitude of the transmitted signal

\section{OFDM SYSTEM}

Orthogonal frequency division multiplexing is a multi-carrier modulation technique in which highrate streams are divided into low-throughput streams in parallel and modulated separately on many closely spaced sub-carriers. The adopted system consists of a tail biting convolutional codes (CC) whose constraint length is 7 in the transmitter side and an RLS adaptive filter in the receiver side. Figure 2 illustrates the system diagram used in our study and the parameters used during the simulation are summarized in Table 2 . 


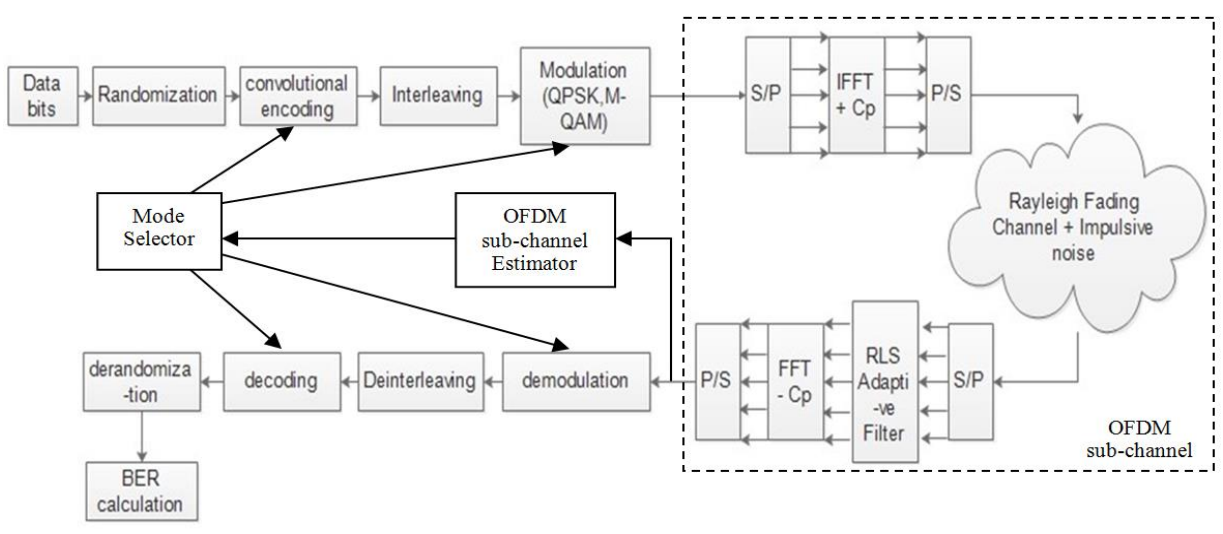

Figure 2. Block diagram of proposed scheme for OFDM system

Table 2. Parameter set of OFDM system simulation

\begin{tabular}{ll}
\hline Parameters & Values \\
\hline Modulation technique & QPSK,M-QAM \\
Number of subcarriers & 16 \\
Size of cyclic prefix & 128 \\
FFT-length & 512 \\
BW & 2 GHZ \\
Channel model & Rayleigh Fading Channel \\
\hline
\end{tabular}

\section{PROPOSED HYBRID TECHNIQUE FOR SaS NOISE SUPPRESSION}

Our solution is to equalize the Rayleigh SaS channel by RLS adaptive filtering and eliminate residual noise in OFDM sub-channels by AMC.

\subsection{RLS adaptive filter}

Adaptive filters are self-design systems that can adapt to different environments so they are used in various applications such as biomedical engineering, active noise control, interference cancellation and so on [23]. Figure 3 represents the concept of interference cancellation. The steps of the RLS filter used in this research are given in [24] and it's summarized as follow:

$$
y(n)=W^{T}(n) x(n)
$$

So, the expression of prediction error $e(n)$ is defined as:

$$
e(n)=d(n)-y(n)
$$

$x(n)$ and $d(n)$ are the primary input and the reference input respectively.

$W(n)$ denotes the coefficient vector (or the weighting vector), where:

$$
\begin{aligned}
& W(n+1)=W(n)+k(n) x(n) \\
& k(n)=\frac{\lambda^{-1} \phi^{-1}(n-1) x(n)}{1+\lambda^{-1} x^{T}(n) \phi^{-1}(n-1) x(n)} \\
& \phi^{-1}(n)=\lambda^{-1} \phi^{-1}(n-1)-\lambda^{-1} k(n) x^{T}(n) \phi^{-1}(n-1)
\end{aligned}
$$

$\phi$ is the cross correlation matrix for $x(n), \phi^{-1}$ is its inverse and $\lambda$ is the forgetting factor.

RLS Adaptive filter coefficients adapt recursively so that the error signal e (n) becomes minimal. Subsequently output signal $\mathrm{y}(\mathrm{n})$ approaches the desired signal. So, the most important part of the impulsive noise is suppressed from the received signal. But, on the other hand, as the minimum error doesn't reach zero, a residual part of noise at the output of the filter still exists. The AMC technique will be used here to to mitigate the effect of this residual noise and improve BER performance. 


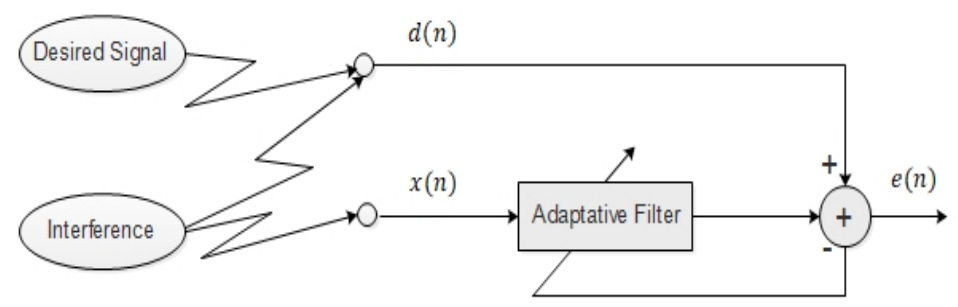

Figure 3. Block diagram of RLS noise concellation

\subsection{Adaptive modulation and convolutionnal coding}

In order to improve the performance of the system Adaptive Modulation and Convolutionnal Coding (AMC) is utilized. AMC technique allows controlling each OFDM sub-channels' constellation size depending on the channel conditions. Data rate, instantaneous BER, channel code/scheme, and constellation size can be controlled using OFDM sub-Channel Quality Information (CQI). This latter is estimated at the receiver side just at the output of the RLS filter. The modulation scheme (BPSK, QPSK, 16-QAM, $64-\mathrm{QAM})$ and convolutional code rates $(1 / 2,2 / 3,3 / 4)$ are adjusted according CQI.

\section{SIMULATION RESULTS}

In this section, we discuss the performance of the proposed technique according to the results of the simulation. The impulsive noise was generated from the PDF [25]. Figure 4 depict the noise generated. The results of the first experiment are given in Figure 5. where we set the length of the adaptive filter $\mathrm{L}=32$ and take different values for the forgetting factor $\lambda$. We can notice that when the value of the forgetting factor increases, the BER decreases. In the second experiment, we used a fixed forgetting factor for different values of the adaptive filter length. From the results presented in Figure 6. It can be seen that the length of the adaptive filter has no significant influence on the BER, contrarily more it increases more the calculation becomes slow. For the rest of the simulations we took $\lambda=0,99999$ and $\mathrm{L}=32$.

Figure 7 presents the BER comparison of uncoded, coded and our proposed hybrid technique. The simulation is done for different $\alpha$ values and according to the results obtained, we notice that the algorithm gives us good results for values of $\alpha$ higher than 1 . Thus, the BER decreases from $1.9 \cdot 10^{-2}$ to $1.8 \cdot 10^{-4}$ for $\alpha=2$ at GSNR of -10 , and from $1.8 \cdot 10^{-3}$ to $1.88 \cdot 10^{-5}$ for $\alpha=1.5$ at GSNR of -4 . Figure 8 shows BER performances of coded and CC-RLS systems for different modulation schemes and convolutional coding (CC) rates. It can be easily deduced that the RLS filter succeeds in reducing impulse noise and improving BER performance for all pairs of modulation patterns. For a very impulsive environment $\alpha=1.2$, the QPSK modulation scheme with $1 / 2 \mathrm{CC}$ rate is best suited. However, for $\alpha=1.8$, the most appropriate modulation scheme is BPSK with $1 / 2 \mathrm{CC}$ rate, in which case the RLS filter yields a gain of about $8 \mathrm{~dB}$ to BER of $2.6 \cdot 10^{-3}$.

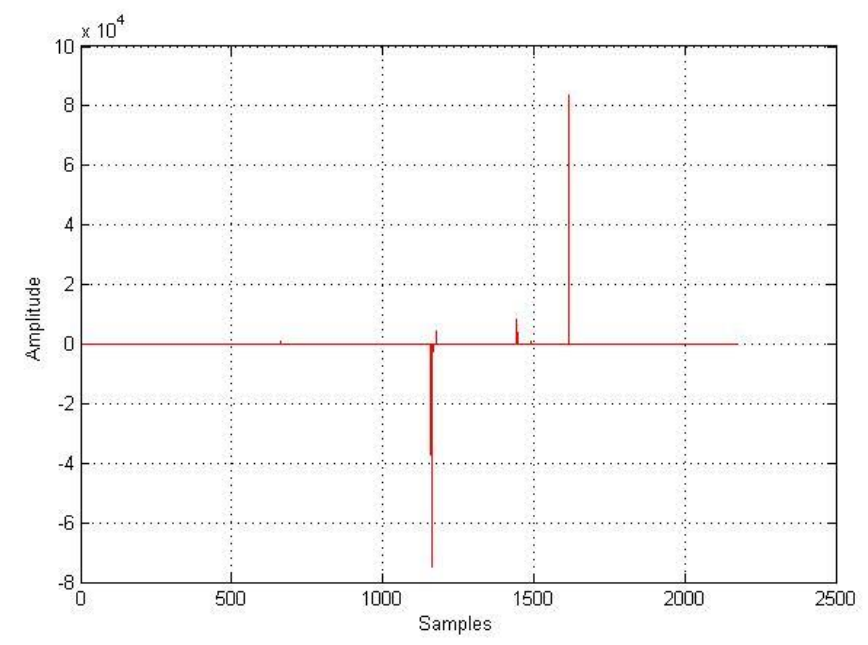

Figure 4. SaS impulsive noise signal 


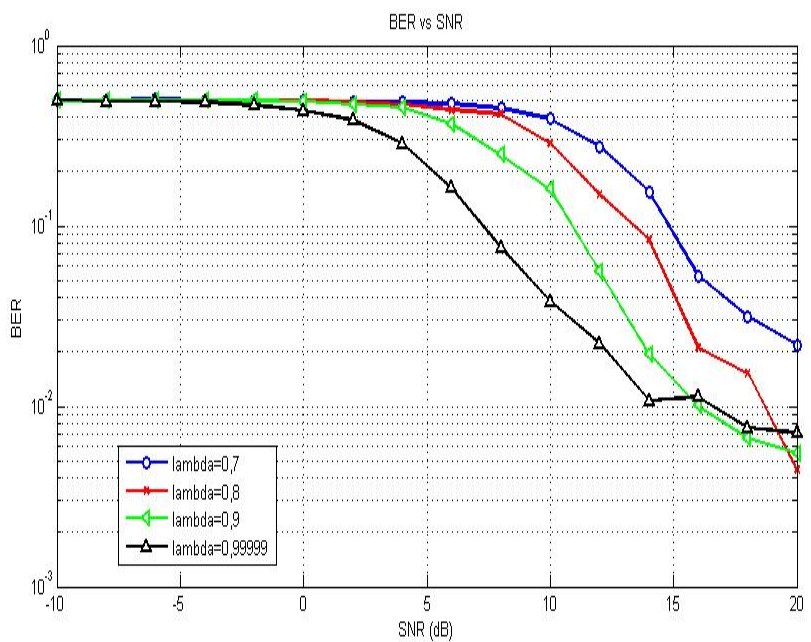

Figure 5. BER vs GSNR with $\mathrm{L}=16$ and differents values of $\lambda$

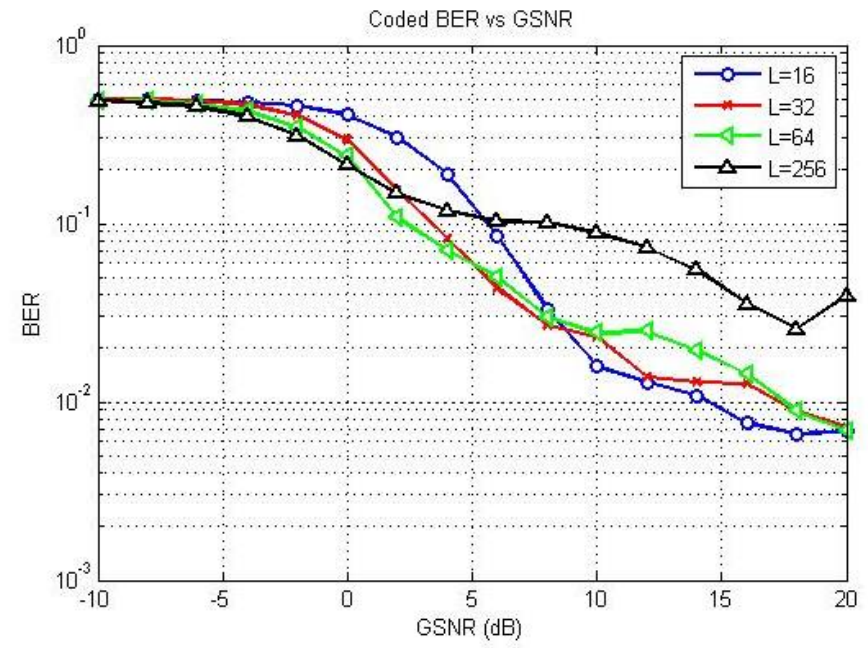

Figure 6. BER vs GSNR with $\lambda=0.99$ and differents values of $\mathrm{L}$
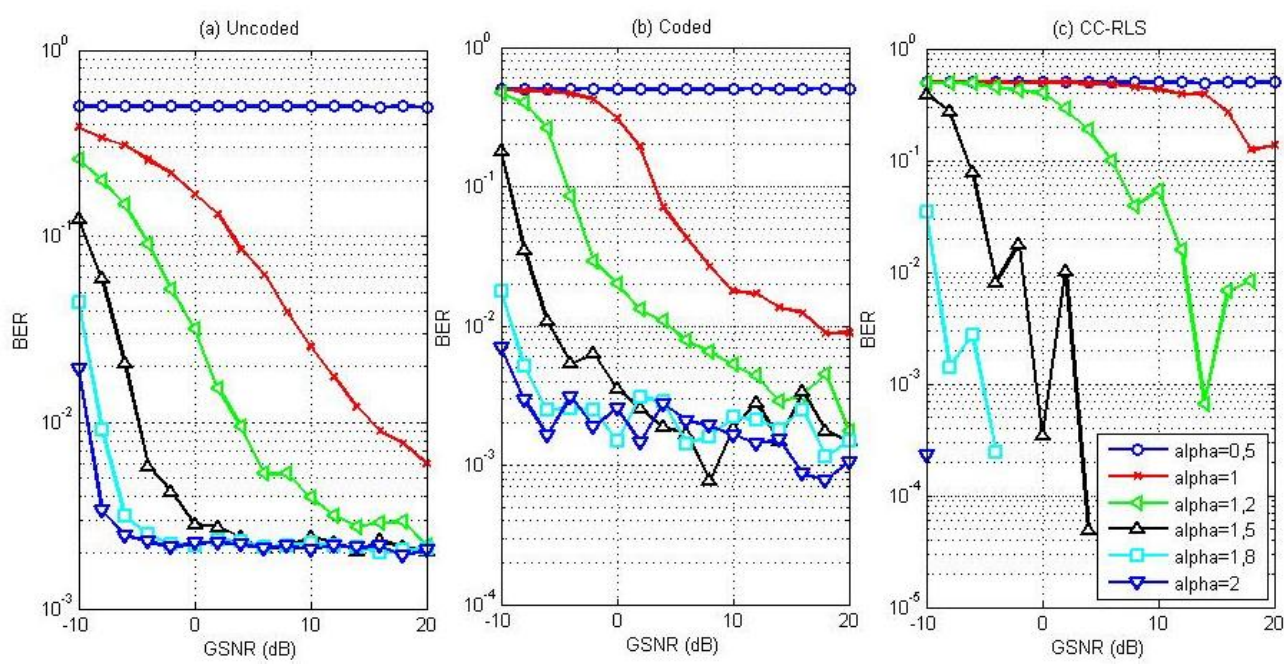

Figure 7. BER comparison of uncoded, coded and the proposed technique for different values of $\alpha$ 

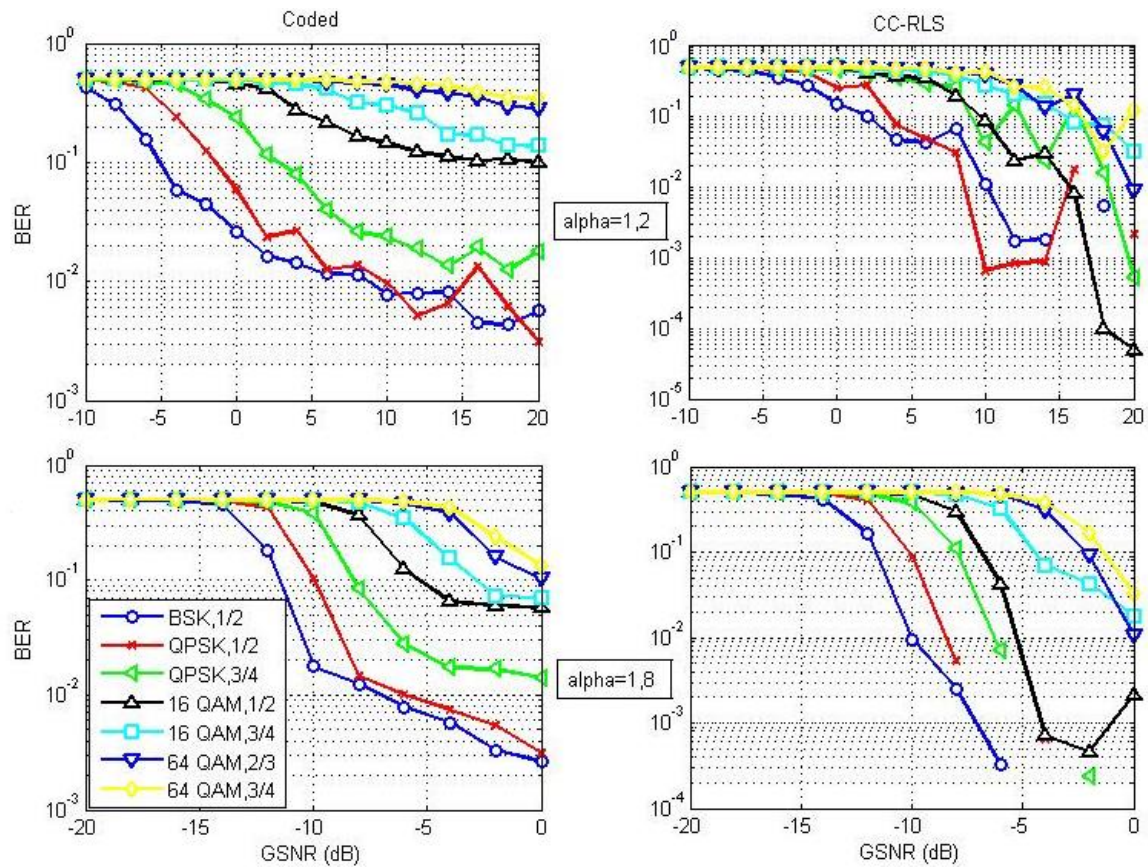

Figure 8. BER performances of coded and CC-RLS systems over different modulation schemes and convolutional coding (CC) rates for $\alpha=1.2$ and $\alpha=1.8$

\section{CONCLUSION}

In this paper, we propose a novel approach for symmetric-alpha-stable $(\mathrm{S} \alpha \mathrm{S})$ noise suppression and BER improvement in OFDM communication systems. We explore the combination of RLS adaptive filters and the AMC technique in an impulsive noise multipath channel environment. The proposed solution equalizes the Rayleigh $\mathrm{S} \alpha \mathrm{S}$ channel by RLS adaptive filtering and eliminates residual noise in OFDM sub-channels by AMC. Simulation results show the effectiveness of our solution for noise cancelation and BER performance improvement even in the case of strong impulsive noise.

\section{REFERENCES}

[1] Barazideh.R., et al.,"Impulsive noise mitigation in underwater acoustic communication systems: experimental studies," IEEE 9th Annual Computing and Communication Workshop and Conference (CCWC), Jan 2019.

[2] Amel Benaissa,. Abdelmalek. A., Feham M., "Improved reliability of power line communication under alphastable noise," 5th International Conference on Electrical Engineering - Boumerdes (ICEE-B), Oct 2017.

[3] Reza., et al., "A nonlinear bayesian filtering framework for ecg denoising," IEEE Transactions on Biomedical Engineering, vol. 54, pp. 2172-2185, 2007.

[4] D. Middleton, "Non-gaussian noise models in signal processing for telecommunications: New methods an results for class a and class b noise models," IEEE Transactions on Information Theory, vol. 45, pp.1129-1149, 1999.

[5] S. P. Herath, "On optimal input distributionandcapacity limit of bernoulli-gaussian impulsive noise channels," IEEE International Conference on Communications (ICC), pp. 3429-3433, 2012.

[6] M. Shao and C. L. Nikias, "Signal processing with fractional lower ordermoments: stable processes and their applications," Proceedings of the IEEE, vol. 81, pp. 986-1010, 1993.

[7] Jean-François Bousquet, et al.,"Antenna array designs for OFDM WLAN indoor transmission," Wireless Pers Commun, vol. 56, pp. 779-789, 2011.

[8] Hwang T., et al., "OFDM and its wireless applications: a survey," IEEE Transactions on Vehicular Technology, vol. 58, pp. 1673-1694, 2009.

[9] Changyoung An and Heung-Gyoon Ryu., "Design and performance comparison of W-OFDM under the nonlinear HPA environment," Wireless Pers Commun, published online: Aug 2017.

[10] G.Berardinelli., et al., "Generalized DFT-spread-OFDM as 5G waveform," IEEE Communications Magazine, vol. 54, pp. 99-105, 2016.

[11] C. An, B. Kim., et al., "Design and evaluation of spectrum efficient WR-OFDM system for $5 G$ and B5G mobile system," IEEE International Conference on Microwaves, Antennas, Communications and Electronic Systems COMCAS, pp. 1-5, 2017.

[12] X. Zhang, et al.,"Filtered-OFDM enabler for flexible waveform in the 5th generation cellular networks," IEEE Globecom, San Diego, CA, 2015. 
[13] S. V. Vaseghi, "Advanced digital signal processing and noise reduction," 355, 4th ed. John Wiley and Sons Ltd, 2000.

[14] Hayat Ullah, et al.,"Wavelet based de-noising using logarithmic shrinkage function," Wireless Pers Commun, vol. 98, pp. 14731488, 2018.

[15] Khedkar, et al.,"Trained adaptive filter based approach to mitigate ICI in OFDM system," Proceedings of International Conference on Pervasive Computing, pp. 1-4, Pune, India, 2015.

[16] S. Mathew and P. Murukan, "Periodic impulsive noise reduction in OFDM based power line communication," International Journal on Research in Engineering and Technology, vol. 3, pp. 517-522, 2014.

[17] Alina Mirza, et al.,"Reduction of impulsive noise in OFDM system using adaptive algorithm," World Academy of Science, Engineering and Technology, International Journal of Computer, Electrical, Automation Control and Information Engineering, vol. 9, 2015.

[18] Srinu Pyla, K. Padma Raju, N. Bala Subrahmanyam, "Performance analysis of adaptive filter channel estimated MIMO OFDM communication system," International Journal of Electrical and Computer Engineering (IJECE), vol. 8(5), pp. 3829-3838, Oct 2018.

[19] M. A. Chitre, et al.,"Optimal and near-optimal signal detection insnapping shrimp dominated ambient noise," IEEE Journalof Oceanic Engineering, vol. 31, pp. 497-503, 2006.

[20] John P. Nolan., "Stable distributions: models for heavy-tailed data", ISBN 0817641599.

[21] V. M. Zolotarev.,"One-dimensional stable distributions," American Mathematical Society, vol. 65, 1986.

[22] Heyem Hamlili, et al., "Convolutional code performance for OFDM system in an alpha-stable noise environment," IEEE International Conference on communications and Electrical Engineering, Algeria, 2018.

[23] Shubhra Dixit and Deepak Nagaria, "LMS adaptive filters for noise cancellation: a review," International Journal of Electrical and Computer Engineering (IJECE), vol.7, pp. 2520-2529, 2017.

[24] Behrouz Farhang-Boroujeny, "Adaptive filters theory and applications," Second Edition, ISBN 978-1-119-97954-8.

[25] Mark Veillette, "Alpha-stable distributions in MATLAB," [Online] Available at: http://math.bu.edu/people/mveillet/html/alphastablepub.html, Accessed: Jul 10, 2018. 